\title{
Spatial aliasing and distortion of energy distribution in the wave vector domain under multi-spacecraft measurements
}

\author{
Y. Narita ${ }^{1}$ and K.-H. Glassmeier ${ }^{1,2}$ \\ ${ }^{1}$ Institut für Geophysik und extraterrestrische Physik, Mendelssohnstr. 3, 38106 Braunschweig, Germany \\ ${ }^{2}$ Max-Planck-Institut für Sonnensystemforschung, Max-Planck-Str. 2, 37191 Katlenburg-Lindau, Germany
}

Received: 7 May 2009 - Revised: 27 July 2009 - Accepted: 27 July 2009 - Published: 5 August 2009

\begin{abstract}
Aliasing is a general problem in the analysis of any measurements that make sampling at discrete points. Sampling in the spatial domain results in a periodic pattern of spectra in the wave vector domain. This effect is called spatial aliasing, and it is of particular importance for multispacecraft measurements in space. We first present the theoretical background of aliasing problems in the frequency domain and generalize it to the wave vector domain, and then present model calculations of spatial aliasing. The model calculations are performed for various configurations of the reciprocal vectors and energy spectra or distribution that are placed at different positions in the wave vector domain, and exhibit two effects on aliasing. One is weak aliasing, in which the true spectrum is distorted because of non-uniform aliasing contributions in the Brillouin zone. It is demonstrated that the energy distribution becomes elongated in the shortest reciprocal lattice vector direction in the wave vector domain. The other effect is strong aliasing, in which aliases have a significant contribution in the Brillouin zone and the energy distribution shows a false peak. These results give a caveat in multi-spacecraft data analysis in that spectral anisotropy obtained by a measurement has in general two origins: (1) natural and physical origins like anisotropy imposed by a mean magnetic field or a flow direction; and (2) aliasing effects that are imposed by the configuration of the measurement array (or the set of reciprocal vectors). This manuscript also discusses a possible method to estimate aliasing contributions in the Brillouin zone based on the measured spectrum and to correct the spectra for aliasing.
\end{abstract}

Keywords. Electromagnetics (Antenna arrays; Signal processing and adaptive antennas) - Space plasma physics (Experimental and mathematical techniques)

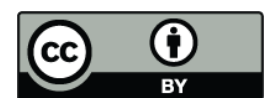

Correspondence to: Y. Narita (y.narita@tu-bs.de)

\section{Introduction}

Recent progress in our understanding of near-Earth space environments such as the solar wind or the Earth's magnetosphere benefits greatly from multi-spacecraft missions that enable us to distinguish between temporal and spatial variations in the observations, and allows us to determine three-dimensional structures in space. The Cluster mission (Escoubet et al., 2001; Balogh et al., 2001) performs fourpoint measurements with its tetrahedron configuration and the THEMIS mission (Angelopoulos, 2008; Auster et al., 2008) performs five point measurements. There exist a number of data analysis methods that are developed particularly for such multi-point measurements, parts of which are summarized in Glassmeier et al. (1995) and Paschmann and Daly $(1998,2008)$. One of the unique and powerful analysis methods in studying dynamics of space plasma is to determine the energy distribution directly in the wave vector domain, referred to as the wave telescope technique (or $k$-filtering) (Neubauer and Glassmeier, 1990; Pinçon and Lefeuvre, 1991; Motschmann et al., 1996; Glassmeier et al., 2001). This technique is based on the minimum variance method of Capon (1969) estimating the energy spectrum or distribution in the frequency and wave vector domain using only several points of measurements.

Energy distributions for fluctuating magnetic field in the wave vector domain have been determined using Cluster and have been presented by several authors such as Glassmeier et al. (2001); Narita et al. (2006, 2007); Sahraoui et al. (2006). Dispersion relations of plasma normal modes can also be determined experimentally from the Cluster data (Narita et al., 2003; Narita and Glassmeier, 2005; Vogt, 2008). Figure 1 displays an example of the energy spectrum in the wave vector domain, observed by Cluster spacecraft in the shock-upstream region. The fluctuation energy is anisotropically distributed between two directions: away from the shock toward the sun $\left(E^{+}\right.$in the spectrum), and

Published by Copernicus Publications on behalf of the European Geosciences Union. 


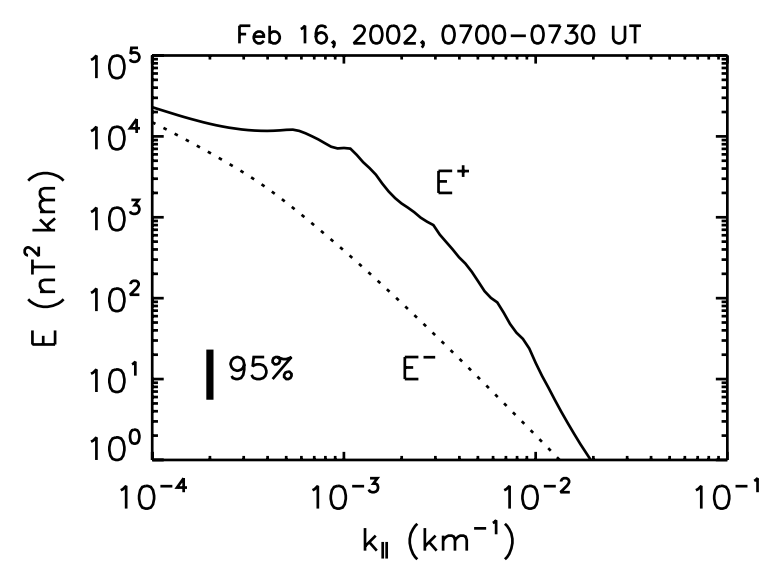

Fig. 1. Example of anisotropic energy spectrum as a function of the wave number parallel to the mean magnetic field, taken from the Cluster observation in the shock-upstream region after Narita et al. (2007). The solid curve represents the spectrum for magnetic field fluctuations in the direction away from the bow shock $\left(E^{+}\right)$ and the dotted curve represents that in the direction toward the bow $\operatorname{shock}\left(E^{-}\right)$.

from the sun toward the shock $\left(E^{-}\right)$. While the larger energy contribution in $E^{+}$can be physically interpreted as the excitation of the waves propagating away from the bow shock, one may also ask how important the aliasing effect is in evaluating the energy spectrum.

It is worthwhile to note that spatial structures can not always be resolved and identified in the multi-point measurements. For example, one can not identify the spatial structure when its characteristic scale (e.g. wavelength) is smaller than the sensor separation distance. In that case the measurement makes under-sampling and the result is subject to aliasing problem. Neubauer and Glassmeier (1990) presented the concept of spatial aliasing under multi-point measurements in space, and measurements using the four Cluster spacecraft indeed suggest the existence of spatial aliasing (Sahraoui et al., 2003; Tjulin et al., 2005).

The configuration of the sensor array can be represented as a set of reciprocal vectors in the wave vector domain, and the analysis in the wave vector domain can be performed within the first Brillouin zone or Wigner-Seitz cell (hereafter referred to as the Brillouin zone or simply the cell) spanned by the half-length reciprocal vectors (Kittel, 1996). Beyond the Brillouin zone the determined spatial structures do not reflect the true structure any more, instead its aliases appears periodically in the wave vector domain.

Aliasing is a general problem not only in science but also in engineering so far as discrete measurements sample a continuous function. The goals of this paper are (1) to generalize aliasing theory from the frequency domain to the wave vector domain, and (2) demonstrate various cases of spatial aliasing in the wave vector domain. It is concluded that the configuration of sensor arrays may significantly influence the measurement of spatial structures due to the aliasing problem even though the analysis is limited within the Brillouin zone. We also discuss a remedy to correct for the aliasing part in the measurement.

\section{Theoretical background}

\subsection{Sampling formulas in time domain}

Sampling formulas, their mathematical foundation, and the aliasing problem have been developed and studied in a number of papers, not only in the celebrated work of Nyquist (1928) and Shannon (1949) but also in recent papers such as Unser (2000) and Kirchner (2005). We briefly discuss the relation between sampling in the time domain and the associated aliasing in the frequency domain. This is essentially an aliasing problem in a one-dimensional system and provides a basis for understanding the aliasing in the wave vector domain.

Spectral aliasing in some under-sampled time series can be understood in the following fashion. Consider a continuous function $g(t)$ in the time domain and its Fourier transform $G(f)$ in the frequency domain. The function $g(t)$ is measured at discrete points, yielding our sampled signal $h(t)$

$h(t)=g(t) \operatorname{III}(t)$,

where $\mathrm{III}(t)$ is called the comb distribution (Bracewell, 2000) which represents an array of delta functions

$\operatorname{III}(t)=\sum_{m=-\infty}^{\infty} \delta\left(f_{0} t-m\right)$.

Here $f_{0}$ denotes the sampling rate $f_{0}=1 / \Delta t$. The comb distribution is a periodic function and can be expressed as a Fourier series

$\mathrm{III}(t)=\sum_{n=-\infty}^{\infty} c_{n} e^{i 2 \pi n f_{0} t}$.

Furthermore, it turns out that the Fourier coefficients $c_{n}$ are all unity:

$c_{n}=\frac{1}{\Delta t} \int_{-\frac{\Delta t}{2}}^{\frac{\Delta t}{2}} \delta\left(f_{0} t\right) e^{-i 2 \pi n f_{0} t} d t=1$,

where one of the properties of the delta function, $\delta\left(f_{0} t\right)=$ $\delta(t) / f_{0}$ was used. The Fourier transform of the sampled signal $h(t)$ is obtained by combining Eqs. (1), (3), and (4).

$$
\begin{aligned}
H(f) & =\int_{-\infty}^{\infty} h(t) e^{-i 2 \pi f_{0} t} d t \\
& =\int_{-\infty}^{\infty} g(t) \operatorname{III}(t) e^{-i 2 \pi f_{0} t} d t \\
& =\sum_{n=-\infty}^{\infty} \int_{-\infty}^{\infty} g(t) e^{-i 2 \pi\left(f-n f_{0}\right) t} d t
\end{aligned}
$$




$$
=\sum_{n=-\infty}^{\infty} G\left(f-n f_{0}\right)
$$

In the last equation one sees the essence of the aliasing problem, that is the Fourier transform of the sampled signal contains not only the Fourier transform of the original function $G(f)$ but also contributions from the $n$-th harmonics of the sampling frequency $G\left(f-n f_{0}\right)$. The power spectrum is proportional to the expectation value of the squared Fourier transform. On the assumption that the phase between any two frequency components is random, the power spectrum is also a sum of the respective alias component,

$$
P(f)=E\left[|H(f)|^{2}\right]=\sum_{n=-\infty}^{\infty}\left|G\left(f-n f_{0}\right)\right|^{2},
$$

where the symbol $E[\cdots]$ denotes the operation of the expectation value. Figure 2 displays the periodic pattern of power spectrum. In this example the contribution from aliases is significant and the true spectrum (top panel) becomes enhanced and distorted in the aliased spectrum (bottom panel) that takes account of the true spectrum and its alias contributions, even within the Nyquist frequency $f_{N}=f_{0} / 2$.

\subsection{Sampling formulas in spatial domain}

The above sampling formulas can be generalized to the spatial domain. Consider now a continuous function of vector in real space, $g(\mathbf{r})$, and its Fourier transform in the wave vector domain, $G(\boldsymbol{k})$. The sampled signal is a filtering of the function $g(\mathbf{r})$ by the set of the delta functions III(r), namely

$h(\mathbf{r})=g(\mathbf{r}) \operatorname{III}(\mathbf{r})$.

The comb distribution in the spatial domain is expressed as

$$
\begin{aligned}
\mathrm{III}(\mathbf{r})= & \sum_{m_{3}=-\infty}^{\infty} \sum_{m_{2}=-\infty}^{\infty} \sum_{m_{1}=-\infty}^{\infty} \delta\left(\frac{\boldsymbol{k}_{3} \cdot \mathbf{r}}{2 \pi}-m_{3}\right) \\
& \delta\left(\frac{\boldsymbol{k}_{2} \cdot \mathbf{r}}{2 \pi}-m_{2}\right) \delta\left(\frac{\boldsymbol{k}_{1} \cdot \mathbf{r}}{2 \pi}-m_{1}\right) \\
= & \sum_{m_{3}=-\infty}^{\infty} \delta\left(\frac{\boldsymbol{k}_{3} \cdot \mathbf{r}}{2 \pi}-m_{3}\right) \sum_{m_{2}=-\infty}^{\infty} \delta\left(\frac{\boldsymbol{k}_{2} \cdot \mathbf{r}}{2 \pi}-m_{2}\right) \\
& \sum_{m_{1}=-\infty}^{\infty} \delta\left(\frac{\boldsymbol{k}_{1} \cdot \mathbf{r}}{2 \pi}-m_{1}\right)
\end{aligned}
$$

or as a Fourier series

$$
\begin{aligned}
\mathrm{III}(\boldsymbol{r}) & =\sum_{n_{3}=-\infty}^{\infty} e^{i n_{3} \boldsymbol{k}_{3} \cdot \mathbf{r}} \sum_{n_{2}=-\infty}^{\infty} e^{i n_{2} \boldsymbol{k}_{2} \cdot \mathbf{r}} \sum_{n_{1}=-\infty}^{\infty} e^{i n_{1} \boldsymbol{k}_{1} \cdot \mathbf{r}} \\
& =\sum_{n_{3}=-\infty}^{\infty} \sum_{n_{2}=-\infty}^{\infty} \sum_{n_{1}=-\infty}^{\infty} e^{i\left(n_{1} \boldsymbol{k}_{1}+n_{2} \boldsymbol{k}_{2}+n_{3} \boldsymbol{k}_{3}\right) \cdot \boldsymbol{r}}
\end{aligned}
$$

where $\boldsymbol{k}_{1}, \boldsymbol{k}_{2}$, and $\boldsymbol{k}_{3}$ are the reciprocal vectors of the primitive vectors of the sensor array (or spacecraft separation vectors) $\boldsymbol{r}_{1}, \boldsymbol{r}_{2}$, and $\boldsymbol{r}_{3}$. Equation (12) is a grouping of the sums

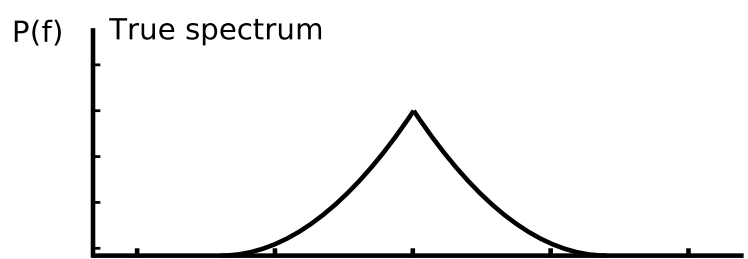

$P(f)$

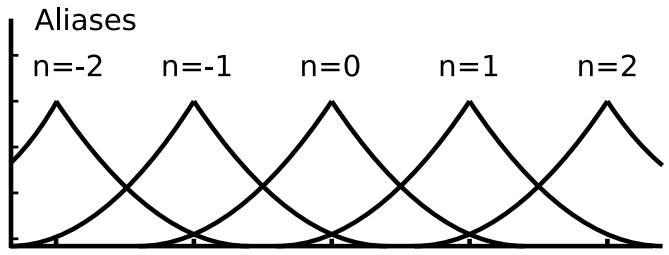

$P(f)$

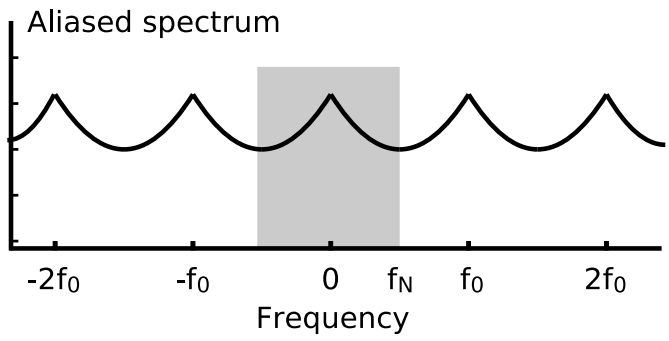

Fig. 2. Illustration of aliasing in the frequency domain. A true spectrum (top panel) appears periodically as aliases in the frequency domain with the frequency shift $n f_{0}$ (middle panel). This results in distortion of the original spectrum (bottom panel) even within the Nyquist frequency $f_{N}$ (shaded area).

in Eq. (11) with respect to $m_{1}, m_{2}$, and $m_{3}$. Equation (13) is obtained by Fourier series expansion of the three delta functions in Eq. (12) (cf. Eqs. 2-4). Equation (14) combines three exponential functions in Eq. (13) together.

The reciprocal vectors are obtained by the primitive vectors $\boldsymbol{r}_{1}, \boldsymbol{r}_{2}$, and $\boldsymbol{r}_{3}$ as

$$
\begin{aligned}
& \boldsymbol{k}_{1}=2 \pi \frac{\boldsymbol{r}_{2} \times \boldsymbol{r}_{3}}{\boldsymbol{r}_{1} \cdot\left(\boldsymbol{r}_{2} \times \boldsymbol{r}_{3}\right)} \\
& \boldsymbol{k}_{2}=2 \pi \frac{\boldsymbol{r}_{3} \times \boldsymbol{r}_{1}}{\boldsymbol{r}_{2} \cdot\left(\boldsymbol{r}_{3} \times \boldsymbol{r}_{1}\right)} \\
& \boldsymbol{k}_{3}=2 \pi \frac{\boldsymbol{r}_{1} \times \boldsymbol{r}_{2}}{\boldsymbol{r}_{3} \cdot\left(\boldsymbol{r}_{1} \times \boldsymbol{r}_{2}\right)},
\end{aligned}
$$

satisfying the orthogonality condition:

$$
\boldsymbol{k}_{i} \cdot \boldsymbol{r}_{j}=2 \pi \delta_{i j}
$$

where $\delta_{i j}$ represents Kronecker's delta. The set of these three reciprocal vectors used in this study are the canonical choice in solid state physics. An alternative set of reciprocal vectors is also in use (Chanteur, 1998). The Fourier transform of $h(\mathbf{r})$ is given as

$H(\boldsymbol{k})=\int h(\mathbf{r}) e^{-i \boldsymbol{k} \cdot \mathbf{r}} d^{3} r$ 


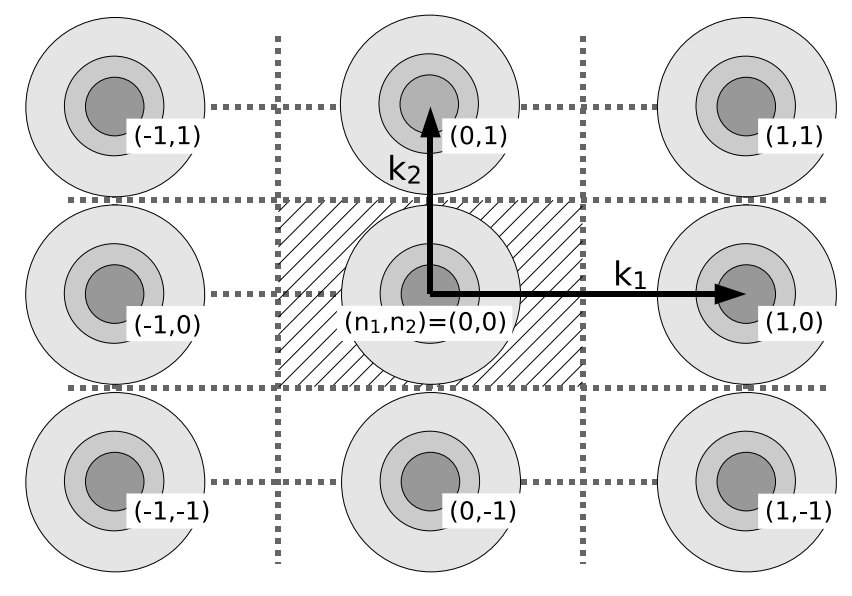

Fig. 3. Aliasing in the wave vector domain. The shaded disks represent the energy distribution with its aliases. The hatched area with dash-lines is the first Brillouin zone (Wigner-Seitz cell) spanned by the half-length reciprocal vectors.

$$
\begin{aligned}
& =\sum_{n_{1}, n_{2}, n_{3}} \int g(\mathbf{r}) e^{-i\left(\boldsymbol{k}-n_{1} \boldsymbol{k}_{1}-n_{2} \boldsymbol{k}_{2}-n_{3} \boldsymbol{k}_{3}\right) \cdot \mathbf{r}} d^{3} r \\
& =\sum_{n_{1}, n_{2}, n_{3}} G\left(\boldsymbol{k}-n_{1} \boldsymbol{k}_{1}-n_{2} \boldsymbol{k}_{2}-n_{3} \boldsymbol{k}_{3}\right)
\end{aligned}
$$

Again on the assumption of random phase, one obtains the spectrum in the wave vector domain as

$$
\begin{aligned}
P(\boldsymbol{k}) & =E\left[|H(\boldsymbol{k})|^{2}\right] \\
& =\sum_{n_{1}, n_{2}, n_{3}}\left|G\left(\boldsymbol{k}-n_{1} \boldsymbol{k}_{1}-n_{2} \boldsymbol{k}_{2}-n_{3} \boldsymbol{k}_{3}\right)\right|^{2}
\end{aligned}
$$

Therefore the spatial sampling results in a periodic structure of the spectrum in the wave vector domain, containing both the true spectrum $|G(\boldsymbol{k})|^{2}$ and its aliases $\mid G\left(\boldsymbol{k}-n_{1} \boldsymbol{k}_{1}-\right.$ $\left.n_{2} \boldsymbol{k}_{2}-n_{3} \boldsymbol{k}_{3}\right)\left.\right|^{2}$. Figure 3 displays an illustration of the spectrum with aliasing in the wave vector domain.

As Neubauer and Glassmeier (1990) state, the periodic structure in the wave vector domain is avoided if we restrict the analysis to a small subvolume of the wave vector space. For example, one can use a sphere in the wave vector domain with the radius equal to the minimum reciprocal vector length, or one can use a right square or cube with this length. It is important that the analysis should be limited to within the Brillouin zone, otherwise the spectrum exhibits a periodic alias pattern in the wave vector domain. However, we stress here that the original spectrum may be enhanced and distorted as shown in Fig. 2 even if we restrict ourselves to the Brillouin zone.

\section{Aliasing in the wave vector domain}

\subsection{An example}

Figure 4 displays an example of aliasing in a twodimensional wave vector domain. The data (the true spectrum) are generated numerically and the associated aliasing effect is studied. The wave vector domain is spanned by the half-length reciprocal vectors $\boldsymbol{k}_{1}=(0.02,0.00) \mathrm{km}^{-1}$ and $\boldsymbol{k}_{2}=(0.00,0.01) \mathrm{km}^{-1}$. The true spectrum (left panel) represents an isotropic energy distribution, centered almost at the origin $\left(k_{x}, k_{y}\right)=(0.0001,0.000) \mathrm{km}^{-1}$ and decaying toward larger wave numbers by a power-law $P(|\boldsymbol{k}|) \propto k^{-\alpha}$ with the index $\alpha=5 / 3$. Contribution from the nearest neighboring aliases with $\left(n_{1}, n_{2}\right)=( \pm 1, \pm 1),(0, \pm 1),( \pm 1,0)$ in the first Brillouin zone is displayed in the middle panel. A large part of the alias contribution comes from the alias $\left(n_{1}, n_{2}\right)=(0,1)$ and $(0,-1)$, since the magnitude of the second reciprocal vector $\boldsymbol{k}_{2}$ is smaller than that of the first one and the alias partners $G\left(\boldsymbol{k}-n_{1} \boldsymbol{k}_{1}-n_{2} \boldsymbol{k}_{2}\right)$ are spaced in the wave vector domain more closely to each other in the direction to $\boldsymbol{k}_{2}$. The right panel in Fig. 4 displays the aliased spectrum which is a sum of the true spectrum (left panel) and the aliased part (middle panel). The aliased spectrum is what we expect to obtain from multi-point measurements. It is interesting that the aliased spectrum does not present an isotropic distribution any more, but is elongated in the direction to $\boldsymbol{k}_{2}$ which is the shortest lattice vector direction in the reciprocal space. It is clear at this stage that the elongation or anisotropy in the energy spectrum or distribution is imposed by the the configuration of the reciprocal vectors, especially how close or different the magnitudes of the vectors $\left|\boldsymbol{k}_{1}\right|$ and $\left|\boldsymbol{k}_{2}\right|$ are from each other. Another effect is that the spectrum is enhanced at various positions in the Brillouin zone. On the other hand, the isotropy in the spectrum is only slightly broken around the peak. The reason for this is that the spectral energy is larger near the peak and furthermore it is at enough distance from the border of the Brillouin zone such that the contribution from the aliases is the smallest there.

Distortion of the spectrum due to aliasing is investigated using two parameters: an enhancement parameter $E$ and an anisotropy parameter $A$. The enhancement parameter is defined as a ratio of the aliased spectrum $P_{\text {als }}$ to the true spectrum $P_{\text {true }}$ as a function of wave number from the spectral peak in a given direction,

$$
E(\boldsymbol{k})=\frac{P_{\text {als }}(\boldsymbol{k})}{P_{\text {true }}(\boldsymbol{k})} .
$$

We choose the maximum and the minimum enhancement direction in this study. Figure 5 displays the enhancement parameter in the example case in these two directions (denoted as "max" and "min" in the aliased spectrum). In the maximum enhancement direction this quantity increases from $E=1.00$ at the spectral peak (which is $k=0.0000 \mathrm{~km}^{-1}$ in Fig. 5) to $E=2.51$ at the largest deviation wave number 


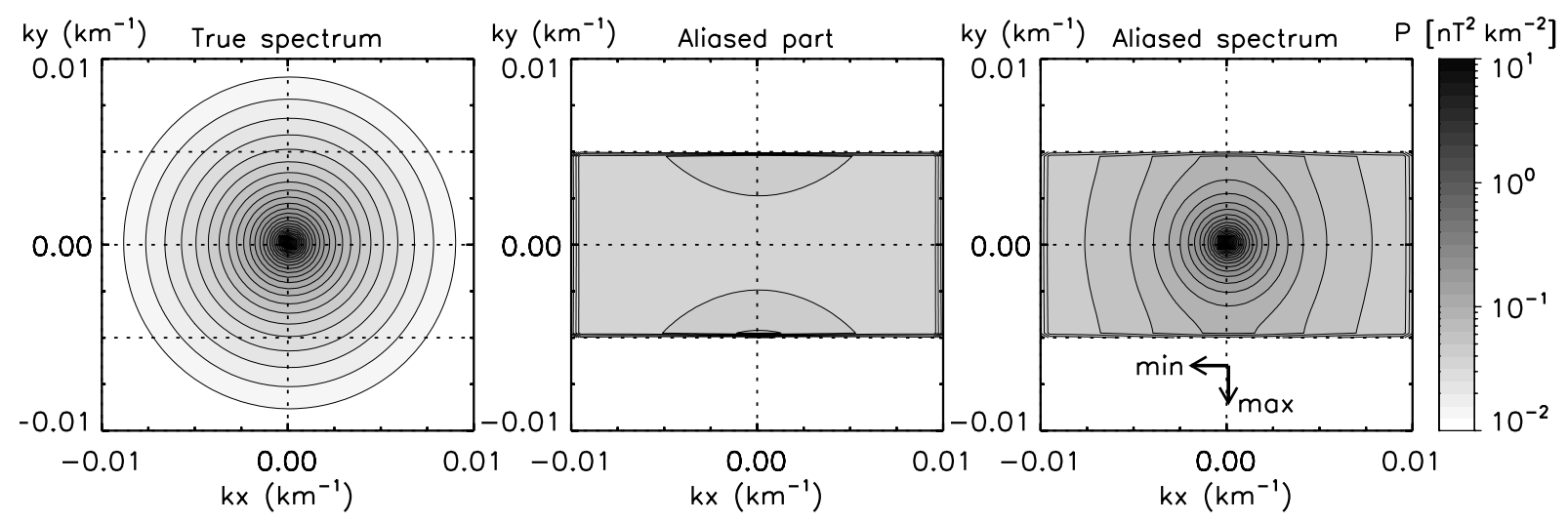

Fig. 4. True spectrum (left), aliased part from the neighboring cells (middle), and aliased spectrum that is a sum of the true spectrum and its aliases (right).

Table 1. Enhancement parameter and anisotropy parameter in the example case.

\begin{tabular}{rccc}
\hline$k\left(\mathrm{~km}^{-1}\right)$ & 0.0000 & 0.0020 & 0.0040 \\
\hline $\max (\mathrm{E})$ & 1.00 & 1.26 & 1.99 \\
$\min (\mathrm{E})$ & 1.00 & 1.25 & 1.77 \\
$\mathrm{~A}(\%)$ & 0 & 2 & 12 \\
\hline
\end{tabular}

$k=0.0048 \mathrm{~km}^{-1}$. In the minimum enhancement direction it also increases but more moderately than in the maximum direction, $E=1.00$ at the spectral peak, $E=2.02$ at $0.0048 \mathrm{~km}^{-1}$, and $E=4.34$ at $0.095 \mathrm{~km}^{-1}$. Some more values of the enhancement parameter are listed in Table 1 and plotted in Fig. 5.

We then investigate the aliased spectrum with the anisotropy parameter that measures a relative difference in the aliased spectrum between the maximum and the minimum enhancement directions:

$A=\frac{\max \left(P_{\mathrm{als}}\right)-\min \left(P_{\mathrm{als}}\right)}{\min \left(P_{\mathrm{als}}\right)}$.

Alternatively one may use the enhancement parameter $E=$ $P_{\text {als }} / P_{\text {true }}$ instead of the aliased spectrum $P_{\text {als }}$ if the true spectrum is isotropic, i.e., $P_{\text {true }}$ is independent from the choice of direction from the spectral peak and only a function of the magnitude of the wave number. We evaluate the anisotropy parameter at two different wave numbers (Table 1 bottom). Anisotropy increases as the wave number becomes larger, up to $A=24 \%$ at the largest wave number available in the maximum enhancement direction, which is $k=0.0048 \mathrm{~km}^{-1}$ (at the right edge of the "max" curve in Fig. 5).

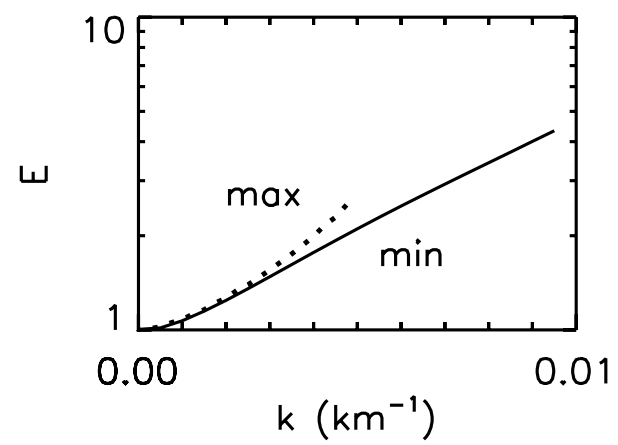

Fig. 5. Enhancement parameter (ratio of aliased to true spectrum) in the maximum (dotted line) and minimum distortion directions (solid line) for the spectrum in Fig. 4. The x-axis measures distance from the peak of the spectrum in the wave number domain.

\subsection{Effect of spectral peak positions and reciprocal vectors}

Here we study how aliasing affects the true spectrum under different conditions: (case 1) the peak position is shifted in the true spectrum; (case 2) the lengths of the reciprocal vectors are changed for the rectangular Brillouin zone; (case 3) the shape of the Brillouin zone is changed from a rectangular to a diamond-shaped cell; and (case 4) the peak position is shifted in the diamond-shaped cell.

\section{Case 1: Peak position}

The effect of the peak position in the Brillouin zone is first investigated. Figure 6 exhibits three cases of the aliased spectrum for the same Brillouin zone configuration as the example case in Fig. 4. The true spectrum is again the same isotropic, power-law distribution but its peak is displaced to different positions in the Brillouin zone, near the origin at 


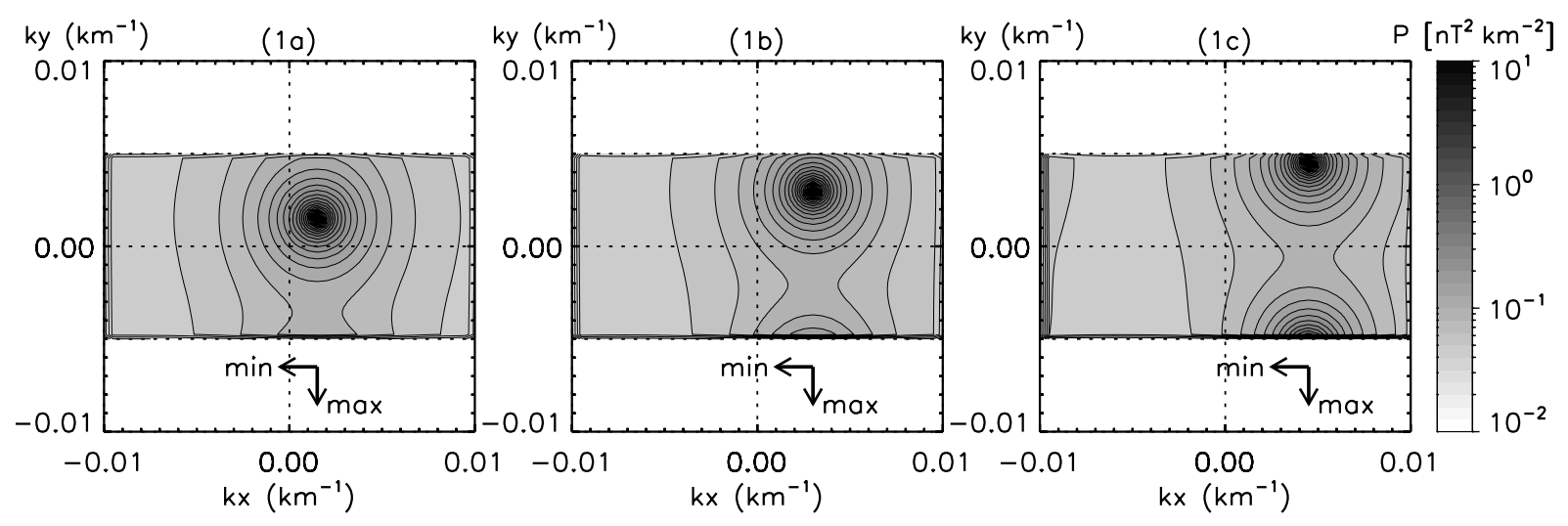

Fig. 6. Aliased spectra in the wave vector domain including both the true and the aliases spectrum for different peak positions of the true spectrum shifted in the Brillouin zone. Peak near the origin (left), at an intermediate distance to the border of the Brillouin zone (middle), near the border (right). Reciprocal vectors are orthogonal to each other and the Brillouin zone forms a rectangular cell.

$\boldsymbol{k}_{c}=(0.0015,0.0015) \mathrm{km}^{-1}$ in case (1a); at an intermediate distance from the origin and the border of the Brillouin zone, $(0.0030,0.0030) \mathrm{km}^{-1}$, in case $(1 \mathrm{~b})$; and almost at the border at $(0.0045,0.0045) \mathrm{km}^{-1}$ in case $(1 \mathrm{c})$.

In all three cases the distribution can be divided into two parts: the distribution is isotropic near the peak, and anisotropic in its surrounding in the sense that the contour lines are elongated and aligned with the shortest lattice vector direction, parallel to $\boldsymbol{k}_{2}$. In case (1a), the distribution exhibits a distortion in the lower half plane in the Brillouin zone, a neck-shaped contour line, due to the alias from $\left(n_{1}, n_{2}\right)=(0,-1)$. In case $(1 \mathrm{~b})$, the peak is shifted closer to the upper border of the Brillouin zone. The top part of the true spectrum is not measurable any more as it is beyond the Brillouin zone. Instead its alias $\left(n_{1}, n_{2}\right)=(0,-1)$ enters the Brillouin zone. This part of the spectrum corresponds to the missing part of the spectrum at the top of the Brillouin zone. In case (1c), the peak is again shifted even closer to the border of the Brillouin zone. The distribution exhibits now two peaks, one at the top of the cell (which reflects the original spectrum) and the other at the bottom which is the alias $\left(n_{1}, n_{2}\right)=(0,-1)$. The transition from the case (1a) to (1c) suggests that the true spectrum is distorted and elongated under a moderate alias contribution, while a false peak appears from the opposite side of the Brillouin zone under strong aliasing. The elongation is roughly aligned with the shortest lattice vector direction, and the distortion of the spectrum is largest where the aliasing contribution is largest in the Brillouin zone.

\section{Case 2: Rectangular cell shape}

Not only the peak position but also the shape of the Brillouin zone is important in the spatial aliasing. Figure 7 compares three different rectangular Brillouin zones and the aliased spectra, while the true spectrum is the same in case case, centered at $\boldsymbol{k}_{c}=(0.0005,0.0005) \mathrm{km}^{-1}$ with the same isotropic, power law distribution as the former examples. The case (2a) exhibits the Brillouin zone spanned by the half-length of reciprocal vectors $\boldsymbol{k}_{1}=(0.020,0.000) \mathrm{km}^{-1}$ and $\boldsymbol{k}_{2}=(0.000,0.016) \mathrm{km}^{-1}$, where the magnitude of the two reciprocal vector is not much different from each other. The measured distribution is almost isotropic. The distortion or elongation of the spectrum can be identified only near the border of the Brillouin zone. The case (2b) exhibits the second reciprocal vector with half-length of the first one, $\boldsymbol{k}_{1}=(0.020,0.000) \mathrm{km}^{-1}$ and $\boldsymbol{k}_{2}=(0.000,0.010) \mathrm{km}^{-1}$. Anisotropy in the aliased spectrum becomes clearer and the distribution is elongated to the shortest lattice vector, in the direction to $\boldsymbol{k}_{2}$. The isotropic distribution can also be identified but its area in the Brillouin zone is smaller than case (2a). The second reciprocal vector is more shortened in case (2c), with $\boldsymbol{k}_{1}=(0.020,0.000) \mathrm{km}^{-1}$ and $\boldsymbol{k}_{2}=(0.000,0.04) \mathrm{km}^{-1}$. The anisotropy is even stronger in this case and the contour lines are almost aligned with the $\boldsymbol{k}_{2}$ direction. The isotropic distribution can be identified only near the peak.

\section{Case 3: Diamond-shaped cell}

Reciprocal vectors are not always mutually orthogonal but have in general various angles. In such a case the Brillouin zone forms a diamond-shaped cell in the wave vector domain. Figure 8 compares the Brillouin zones with three different angles of the reciprocal vectors: $50^{\circ}(3 \mathrm{a}), 30^{\circ}(3 \mathrm{~b})$, and $20^{\circ}(3 \mathrm{c})$. Again, the true spectrum is the same as the rectangular cell case in Fig. 7, isotropic, power law distribution with $\alpha=5 / 3$ centered at $\boldsymbol{k}_{c}=(0.0005,0.0005) \mathrm{km}^{-1}$. The reciprocal vectors in case (3a)-(3c) have all the same magnitude. In case (3a) the Brillouin zone is spanned by the halflength of the reciprocal vectors $\boldsymbol{k}_{1}=(0.0147,0.0053) \mathrm{km}^{-1}$ an $\boldsymbol{k}_{2}=(0.0053,0.0147) \mathrm{km}^{-1}$ with the mutual angle $50^{\circ}$. The distribution is only moderately distorted and almost 


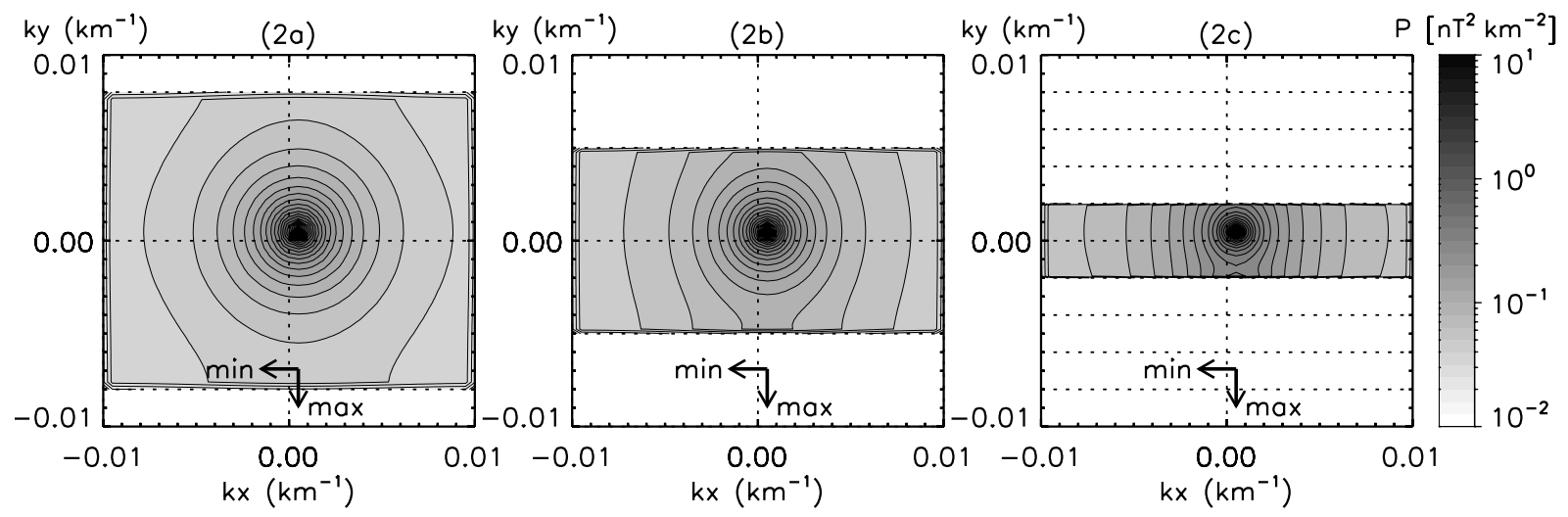

Fig. 7. Aliased spectra for different rectangular cell shapes of the Brillouin zone, close to right square (left), moderately narrow in the vertical reciprocal vector length (middle), even narrower in that length (right). The dotted lines are parallel to the reciprocal vectors.

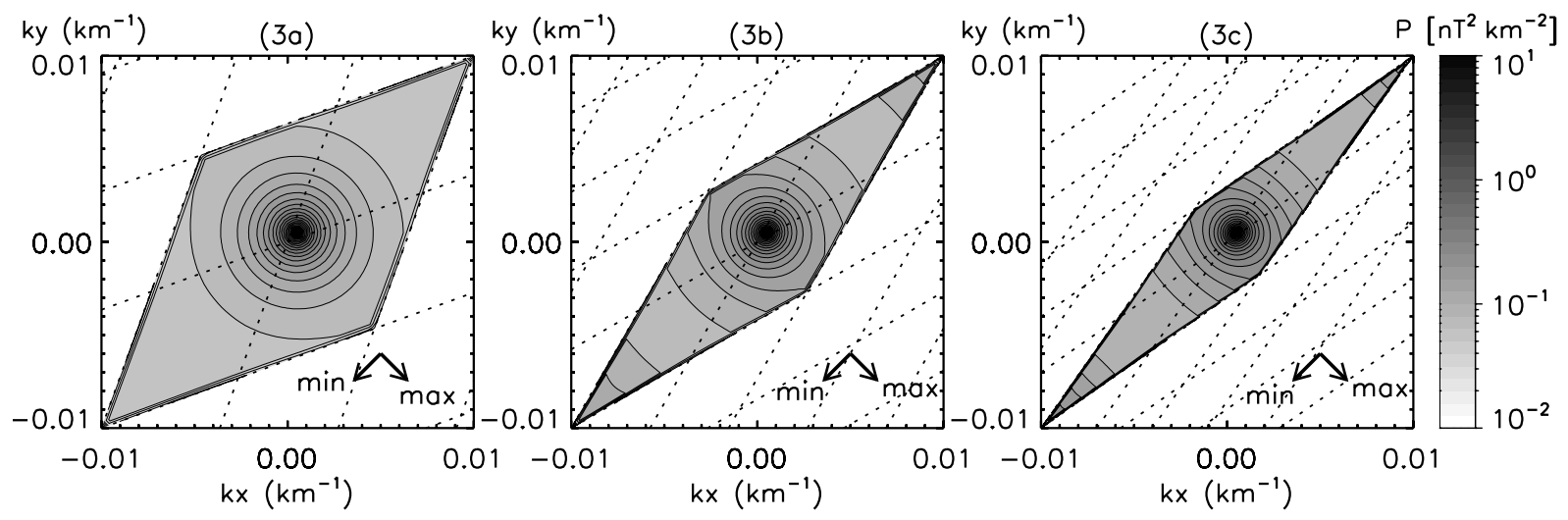

Fig. 8. Aliased spectra for different diamond-shaped Brillouin zones, with the angle between the two reciprocal vectors $50^{\circ}\left(\right.$ left), $30^{\circ}$ (middle), and $20^{\circ}$ (right).

isotropic except for the region near the corner of the Brillouin zone. The distribution is weakly elongated in the direction to the left-top and right-bottom corners which represents the shortest lattice vector direction, $-\boldsymbol{k}_{1}+\boldsymbol{k}_{2}$ (toward the lefttop corner of the cell) or $-\boldsymbol{k}_{1}+\boldsymbol{k}_{2}$ (toward the right-bottom corner). Case ( $3 \mathrm{~b})$ exhibits the mutual angle $30^{\circ}$ between the two reciprocal vectors, $\boldsymbol{k}_{1}=(0.0127,0.0073) \mathrm{km}^{-1}$ an $\boldsymbol{k}_{2}=(0.0073,0.0127) \mathrm{km}^{-1}$. The area of the isotropic distribution becomes smaller and the contour line are more aligned with the shortest lattice vector direction. Aliasing contribution can also be found near the right-top and the left-bottom corners (in the direction to longest lattice vector directions, $\boldsymbol{k}_{1}+\boldsymbol{k}_{2}$ ) and the spectral energy increases near these corners. Case (3c) exhibits even narrower angle, $20^{\circ}$ with $\boldsymbol{k}_{1}=$ $(0.0118,0.0082) \mathrm{km}^{-1}$ and $\boldsymbol{k}_{2}=(0.0082,0.0118) \mathrm{km}^{-1}$. The isotropic distribution area is again small and the distribution is overall anisotropic, though the elongation of the distribution is not very much different from case (3b). At the right-top and the left-bottom corners the distribution exhibits an enhanced contribution from the aliases such that the spectral energy first decreases and then increases near the corner of the cell in the longest lattice vector directions.

\section{Case 4: Peak position in diamond-shaped cell}

Peak position of the true spectrum is shifted in the diamondshaped Brillouin zone with the angle $30^{\circ}$, the same configuration as Fig. 8 middle panel. The shift direction is toward the upper border of the cell. Figure 9 compares the peak position shifted to the upper side of the Brillouin zone, from $\boldsymbol{k}_{c}=(0.0005,0.0010) \mathrm{km}^{-1}$ in case (4a), to $(0.0005,0.0020) \mathrm{km}^{-1}$ in case $(4 \mathrm{~b})$, and then to $(0.0005,0.0030) \mathrm{km}^{-1}$ in case $(4 \mathrm{c})$. In case (4a) the isotropic distribution can be seen only near the peak, otherwise the overall distribution is moderately anisotropic and elongated to the shortest lattice vector direction $\left(-\boldsymbol{k}_{1}+\boldsymbol{k}_{2}\right)$. In case ( $4 b)$ the distribution has again a small isotropic region near the peak and an elongated structure in its surrounding. 


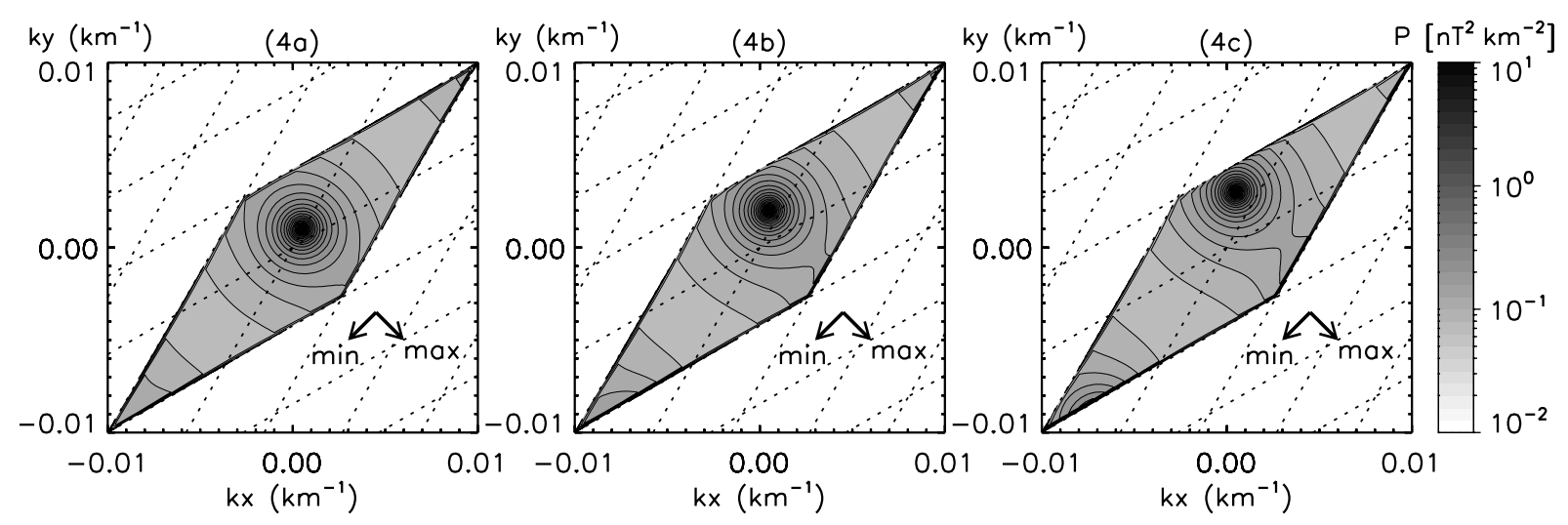

Fig. 9. Aliased spectra for different peak positions in a diamond-shaped Brillouin zone. The peak is shifted to the top side of the Brillouin zone. Peak close to the origin in the cell (left), between the origin and the border of the cell (middle), and near the border (right).
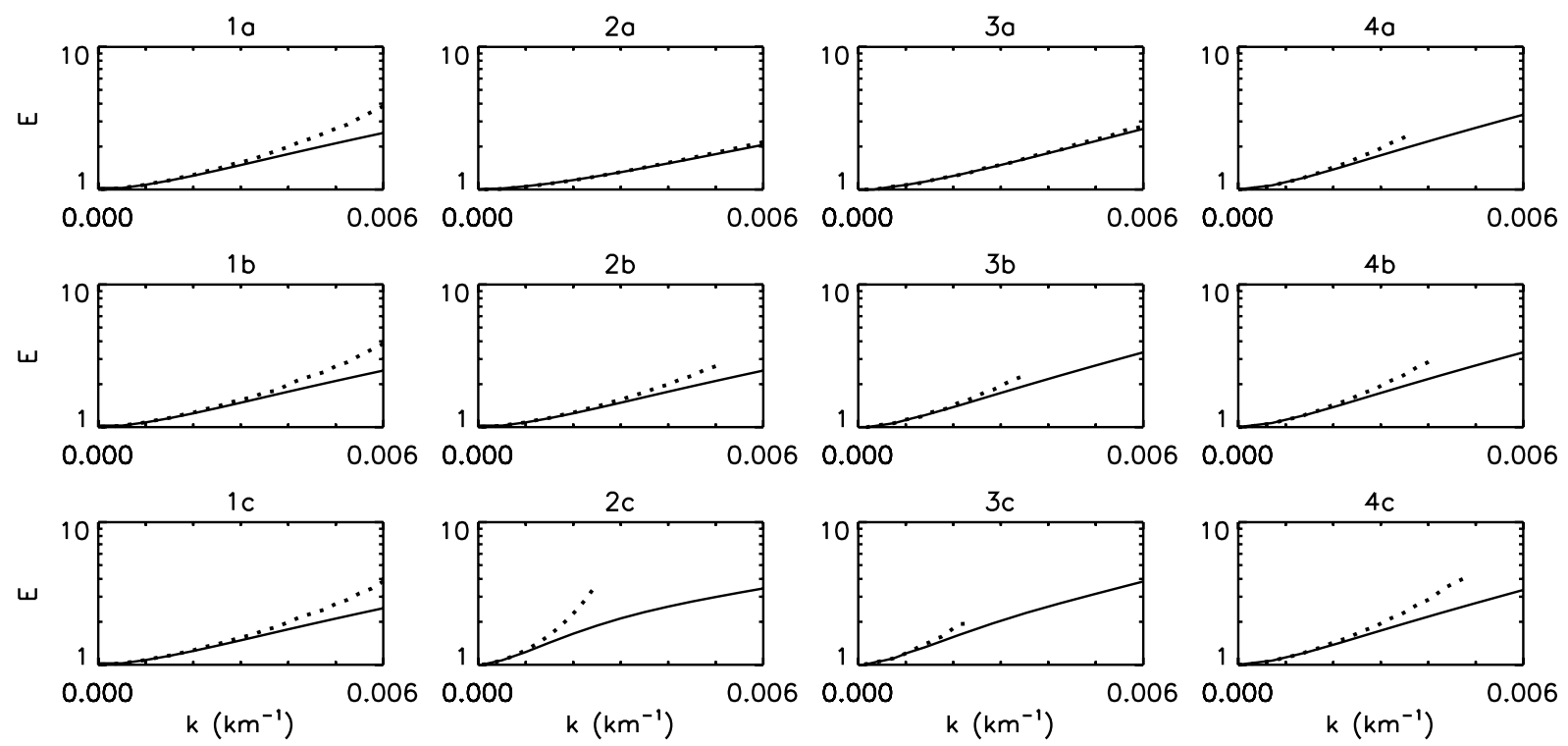

Fig. 10. Enhancement parameter in the maximum (dotted lines) and the minimum distortion directions (solid lines) for the twelve spectra presented in Figs. 6 to 9.

There is an asymmetry in the distribution between the righttop and left-bottom corners. The distorted distribution at the left-bottom corner comes from the alias $\left(n_{1}, n_{2}\right)=(0,-1)$. In case (4c) this alias enters more in the Brillouin zone and two populations are identified in the distribution, the original peak at the top of the cell and its alias at the left-bottom corner. The distribution is isotropic near these peaks, otherwise the contour lines are elongated to the left-top corner direction.

\subsection{Quantitative analysis}

The enhancement parameter $E$ and the anisotropy parameter $A$ are determined for the twelve spectra (1a) to (4c) to quantify the effect of aliasing. The enhancement parameter in each case is summarized in Table 2 at three distinct wave numbers from the spectral peak, and is also plotted in Fig. 10.

\section{Case 1}

All the three spectra (1a), (1b), and (1c) exhibit very similar enhancement: an increase from $E=1.00$ at the peak $(k=$ $0.0000 \mathrm{~km}^{-1}$ ) to $E=3.85$ at $k=0.0060 \mathrm{~km}^{-1}$ in the maximum direction, and also an increase from from $E=1.00$ to $E=2.49$ in the minimum direction. The anisotropy parameter increases from $A=0 \%$ at $0.0020 \mathrm{~km}^{-1}$ to $A=12 \%$ at $0.0040 \mathrm{~km}^{-1}$ and to $A=55 \%$ at $0.0060 \mathrm{~km}^{-1}$ in the three cases. The anisotropy parameter at $0.0060 \mathrm{~km}^{-1}$ is plotted 
Table 2. Enhancement parameter for the twelve spectra in Figs. 6 to 9 in the maximum and the minimum distortion directions.

\begin{tabular}{rccc}
\hline $\mathrm{k}\left(\mathrm{km}^{-1}\right)$ & 0.002 & 0.004 & 0.006 \\
\hline case 1a $\max (\mathrm{E})$ & 1.26 & 1.99 & 3.85 \\
$\min (\mathrm{E})$ & 1.25 & 1.77 & 2.49 \\
$1 \mathrm{~b} \max (\mathrm{E})$ & 1.26 & 1.99 & 3.85 \\
$\min (\mathrm{E})$ & 1.25 & 1.77 & 2.49 \\
1c $\max (\mathrm{E})$ & 1.26 & 1.99 & 3.85 \\
$\min (\mathrm{E})$ & 1.25 & 1.77 & 2.49 \\
case $2 \mathrm{a} \mathrm{max}(\mathrm{E})$ & 1.16 & 1.54 & 2.15 \\
$\min (\mathrm{E})$ & 1.16 & 1.53 & 2.06 \\
$2 \mathrm{~b} \max (\mathrm{E})$ & 1.26 & 1.99 & - \\
$\min (\mathrm{E})$ & 1.25 & 1.77 & 2.49 \\
$2 \mathrm{c} \max (\mathrm{E})$ & 2.29 & - & - \\
$\min (\mathrm{E})$ & 1.65 & 2.56 & 3.43 \\
case 3a max(E) & 1.24 & 1.82 & 2.74 \\
$\min (\mathrm{E})$ & 1.24 & 1.80 & 2.66 \\
$3 \mathrm{~b} \max (\mathrm{E})$ & 1.43 & - & - \\
$\min (\mathrm{E})$ & 1.38 & 2.18 & 3.35 \\
$3 \mathrm{c} \max (\mathrm{E})$ & 1.81 & - & - \\
$\min (\mathrm{E})$ & 1.57 & 2.56 & 3.84 \\
$\min (\mathrm{E})$ & 1.38 & 2.18 & 3.35 \\
case 4a $\max (\mathrm{E})$ & 1.43 & - & - \\
4b $\max (\mathrm{E})$ & 1.43 & 2.86 & - \\
$\min (\mathrm{E})$ & 1.38 & 2.18 & 3.35 \\
4c $\max (\mathrm{E})$ & 1.43 & 2.86 & - \\
$\min (\mathrm{E})$ & 1.38 & 2.18 & 3.35 \\
\hline & & &
\end{tabular}

in Fig. 11 (left top) as a function of $\kappa$ which is a relative closeness of the spectral peak to the boundary of the Brillouin zone,

$\kappa=\left|\frac{\boldsymbol{k}_{c}-\boldsymbol{k}_{b}}{\boldsymbol{k}_{b}}\right|$.

Here $\boldsymbol{k}_{b}$ denotes the boundary wave vector of the Brillouin zone closest from the spectral peak $\boldsymbol{k}_{c}$.

\section{Case 2}

In case (2a) the enhancement parameter almost degenerates between the maximum and the minimum directions, and it increases from $E=1.00$ at the peak to $E=2.15$ (maximum) and $E=2.06$ (minimum) at the wave number $0.0060 \mathrm{~km}^{-1}$. Degenerated enhancement curves are a sign of almost isotropic distribution, and in fact the anisotropy parameter is $A=4 \%$ at $0.0060 \mathrm{~km}^{-1}$. In case (2b) the maximum enhancement increases from $E=1.00$ at the spectral peak to $E=2.7$ at $0.0050 \mathrm{~km}^{-1}$. The largest wave number in the maximum direction is diminished, reflecting the fact that the second reciprocal vector $\boldsymbol{k}_{2}$ is shorter than that in case (2a). The minimum enhancement in (2b) increases from $E=1.00$ at the peak to $E=2.49$ at $0.0060 \mathrm{~km}^{-1}$. In case $(2 c)$ the wave number range for the maximum enhancement is even shorter, and varies from $E=1.00$ at the

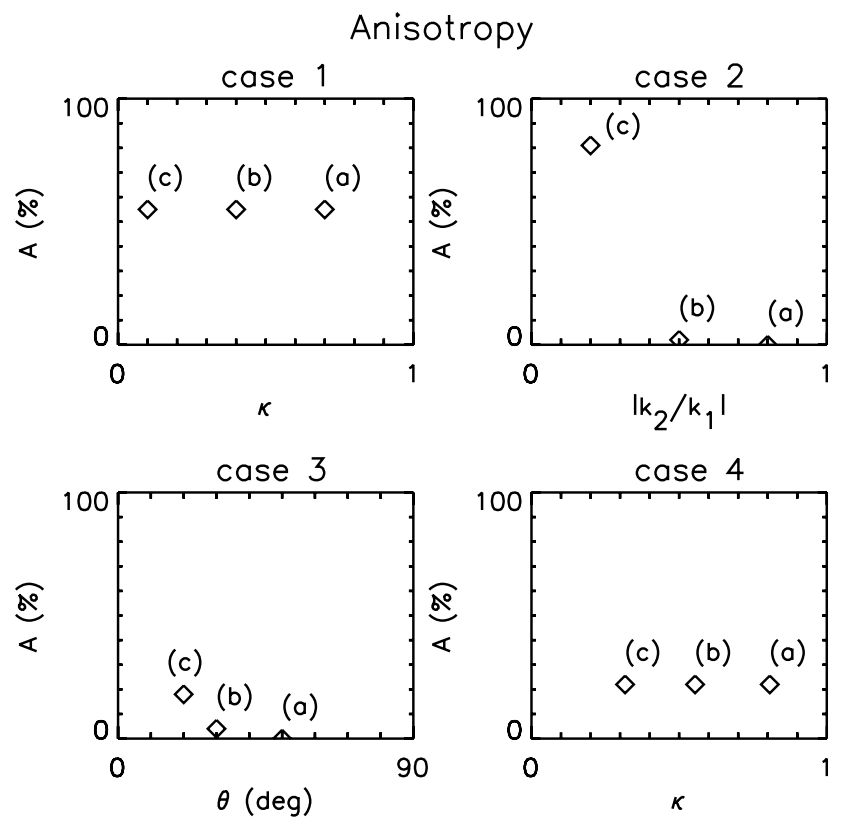

Fig. 11. Anisotropy parameter as a function of the closeness $\kappa$ of the spectral peak to the boundary of the Brillouin zone measured at $k=0.0060 \mathrm{~km}^{-1}$ from the spectral peak (case 1), the ratio of the two reciprocal vector magnitudes at $k=0.0024 \mathrm{~km}^{-1}$ (case 2), the angle between the two reciprocal vectors at $k=0.0022 \mathrm{~km}^{-1}$ (case 3 ), and the peak-boundary closeness $\kappa$ at $k=0.0036 \mathrm{~km}^{-1}$ (case 4).

peak to $E=3.33$ at $0.0024 \mathrm{~km}^{-1}$, whereas the minimum enhancement varies from $E=1.00$ at the peak to $E=1.84$ at $0.0024 \mathrm{~km}^{-1}$ and to $E=3.43$ at $0.0060 \mathrm{~km}^{-1}$. The anisotropy parameter measured at $k=0.0020 \mathrm{~km}^{-1}$ is $A=$ $0 \%(2 \mathrm{a}), 1 \%(2 \mathrm{~b})$, and $38 \%(2 \mathrm{c})$. The anisotropy parameter increases more rapidly from $(2 \mathrm{a})$ to $(2 \mathrm{c})$ when measured at a larger wave number. Figure 11 displays the anisotropy parameter $4 \%$ (2a) to $29 \%$ (2b) and to $81 \%$ (2c) measured at $k=0.0024 \mathrm{~km}^{-1}$ (the maximum wave number available in case (2c)) as a function of the ratio of the two reciprocal vectors $\left|\boldsymbol{k}_{2} / \boldsymbol{k}_{1}\right|$. Anisotropy becomes stronger for a shorter second reciprocal vector $\boldsymbol{k}_{2}$.

\section{Case 3}

In case (3a) the enhancement again almost degenerates and varies from $E=1.00$ at the spectral peak $k=$ $0.0000 \mathrm{~km}^{-1}$ to $E=1.24$ (both maximum and minimum) at $k=0.0020 \mathrm{~km}^{-1}$, and to $E=2.74$ (maximum) and $E=2.66$ (minimum) at $k=0.0060 \mathrm{~km}^{-1}$. In case (3b) the maximum enhancement varies from $E=1.00$ to $E=1.43$ at $k=0.0020 \mathrm{~km}^{-1}$, and to $E=2.24$ at $k=0.0034 \mathrm{~km}^{-1}$. The minimum enhancement varies from $E=1.00$ to $E=1.38$ at $0.0020 \mathrm{~km}^{-1}$, and to $E=1.90$ at $k=0.0034 \mathrm{~km}^{-1}$. In case (3c) the maximum enhancement varies from $E=1.00$ to $E=$ 1.81 at $0.0020 \mathrm{~km}^{-1}$. The minimum enhancement varies 


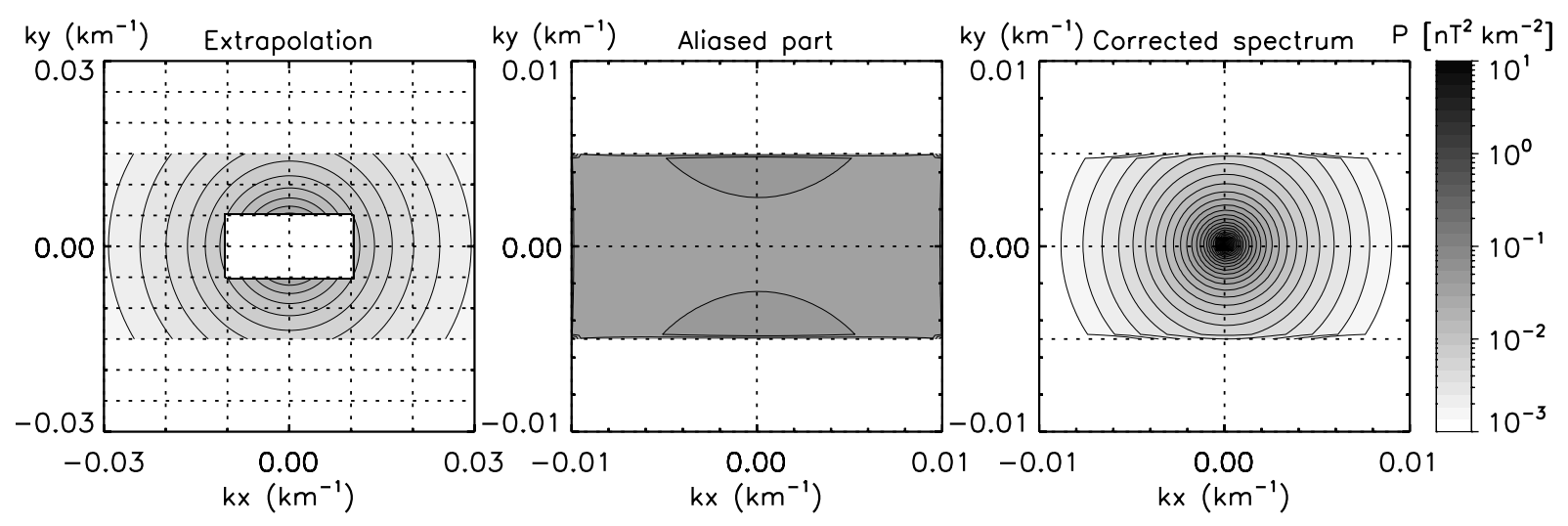

Fig. 12. Reconstruction of the spectrum by correcting the aliases. The spectrum is extrapolated for the neighboring cells in the wave vector domain (left panel), and then the alias of the extrapolated parts are estimated in the Brillouin zone (middle panel). The corrected or reconstructed spectrum is displayed in the right panel.

from $E=1.00$ to $E=1.57$ at $0.0020 \mathrm{~km}^{-1}$. The anisotropy parameter increases moderately toward larger wave numbers in each case. For example, case (3a) exhibits the anisotropy parameter $A=0 \%, 1 \%$, and to $3 \%$ at $k=0.0020 \mathrm{~km}^{-1}$, $0.0040 \mathrm{~km}^{-1}$, and $0.0060 \mathrm{~km}^{-1}$, respectively. Anisotropy increases a little more when the angle between the two reciprocal vectors becomes narrower when measured at a fixed wave number. The anisotropy parameter is plotted as a function of the angle between the two reciprocal vectors in Fig. 11. Anisotropy increases for smaller angles of reciprocal vectors, and it varies from $0 \%$ (3a) to $4 \%$ (3b) and to $18 \%$ (3c) at $k=0.0022 \mathrm{~km}^{-1}$ (largest wave number available in (3c)).

\section{Case 4}

Wave number range in the maximum enhancement direction is short in (4a) and largest in (4c), but the three cases do not exhibit any significant difference in the enhancement parameter. The maximum enhancement parameter increases from $E=1.00$ at the spectral peak to $E=1.43$ at $k=0.0020 \mathrm{~km}^{-1}$ in (4a), (4b), and (4c), and then to $E=2.86$ at $k=0.0040 \mathrm{~km}^{-1}$ in (4b) and (4c). The enhancement parameter in the minimum direction increases from $E=1.00$ at the peak to $E=1.38$ at $k=0.0020 \mathrm{~km}^{-1}, E=2.18$ at $k=0.0040 \mathrm{~km}^{-1}$, and $E=3.35$ at $k=0.0060 \mathrm{~km}^{-1}$ in (4a) to (4c). The anisotropy parameter is $A=4 \%$ in the three cases when measured at $=0.0020 \mathrm{~km}^{-1}$. Figure 11 plots the anisotropy parameter measured at $=0.0036 \mathrm{~km}^{-1}$ (largest wave number available in (4a)) as a function of $\kappa$, the closeness of the spectral peak to the boundary of the Brillouin zone.

\section{Correction of spatial aliasing}

The procedure of correcting the spectrum for aliasing effect is mathematically to estimate in Eq. (9) or (23) the function $G$ for given function $P$. There is, however, an uncertainty in this inverted-procedure because the true spectrum $G$ must first be known to estimate the contribution of the aliases. The correction can in principle be achieved by an iteration scheme, that is to guess a true spectrum and then estimate aliased spectrum using the reciprocal vectors and the assumed true spectrum. If the guessed spectrum yields an aliased spectrum which is close to the measured one, then the guessed spectrum can be used to correct the spectrum by subtracting the aliased part from the measured spectrum. If the guessed spectrum yields a different aliased spectrum from the measured one, then we modify the guessed spectrum and repeat fitting procedure of the measured spectrum until the guessed spectrum provides a reasonably close spectrum to the measurement.

One of the ways to guess a true spectrum is to investigate the measured spectrum near its peak. As the quantitative analysis suggests, aliasing effect is smallest in this region and the spectrum near the peak can potentially be used for an extrapolation of the energy distribution in the area exterior of the Brillouin zone. Aliased spectrum is then estimated based on the extrapolated spectrum, and the measured spectrum is corrected for the aliases. Figure $12 \mathrm{em}-$ ploys this concept and applies the correction method to the example case of Fig. 4. The extrapolation is performed for the core distribution in the neighboring cells of the Brillouin zone (Fig. 12 left), where the peak and the power-law index for the core distribution are determined in the measured distribution by a fitting procedure. The extrapolated distribution is displaced to the neighboring reciprocal lattice points (aliasing at $n_{1}= \pm 1, n_{2}= \pm 1$ ), and the total contribution from these aliases is estimated in the Brillouin zone (Fig. 12 
middle). The measured spectrum is then finally corrected for the aliases (Fig. 12 right).

Our quantitative analysis suggests that anisotropy induced by spatial aliasing is small, say a few $\%$, near the spectral peak under $20 \%$ of the longer reciprocal vector magnitude $\left|\boldsymbol{k}_{1}\right|$, except for a small ratio between the two reciprocal vectors $\left|\boldsymbol{k}_{2} / \boldsymbol{k}_{1}\right|<0.5$ or a small angle of the two vectors $\theta<30^{\circ}$. Wave telescope technique developed for multi-spacecraft measurements (Pinçon and Lefeuvre, 1991; Motschmann et al., 1996; Glassmeier et al., 2001) provides the wave vector resolution better than $1 \%$ of the reciprocal vector (see Fig. 1 ranging over two orders of magnitude of wave number) and such a wave analysis technique is capable of resolving the spectrum near its peak for correction of aliasing effect. Of course, there are always uncertainties in the correction procedure, in guessing the true spectrum, for example, if it is isotropic or anisotropic, or power-law or Gaussian curve, or to which order the aliases are taken into account (how many alias partners to be counted).

\section{Conclusions}

Aliasing is a general problem whenever discrete measurements sample continuous quantities. Periodic sampling of a continuous function results in a periodic structure in the Fourier domain. In this paper we have generalized aliasing in the frequency domain to the wave vector domain, and shown that this spatial aliasing not only distorts the true spectrum but also enters directly in the Brillouin zone. Aliasing in the wave vector domain has two effects, as investigated numerically in Sect. 3. One is weak aliasing, which yields anisotropy and asymmetry in the distribution. The distortion represents primarily elongation of the distribution in the shortest lattice vector direction. Weak aliasing is determined by the configuration of the reciprocal vectors and also by the peak position of the energy distribution in the Brillouin zone. Another effect is strong aliasing, in which the spectral energy becomes dominated by the alias in some parts of the Brillouin zone. The distribution exhibits several peaks (original peak and aliased peak) in the case of strong aliasing. A possible method for correcting the spectrum for aliasing is also discussed. Using several assumptions the true spectrum can be recovered from the measured spectrum by estimating the aliasing contribution in the Brillouin zone. We conclude our study with a conjecture that if the correction method is applied to the wave telescope technique for real spacecraft data, spatial structures of the solar wind, the bow shock, and the magnetosphere will be more precisely determined.

Acknowledgements. This work was financially supported by Bundesministerium für Wirtschaft und Technologie and Deutsches Zentrum für Luft- und Raumfahrt, Germany, under contract 50 OC 0901. Discussions with M. L. Goldstein and F. Sahraoui are gratefully acknowledged.
Topical Editor I. A. Daglis thanks J. Vogt and another anonymous referee for their help in evaluating this paper.

\section{References}

Angelopoulos, V.: The THEMIS Mission, Space Sci. Rev., 141, 534, 2008.

Auster, H.-U., Glassmeier, K.-H., Magnes, W., Aydogar, O., Baumjohann, W., Constantinescu, D., Fischer, D., Fornaçon, K.H., Georgescu, E., Harvey, P., Hillenmaier, O., Kroth, R., Ludlam, M., Narita, Y., Nakamura, R., Okrafka, K., Plaschke, F., Richter, I., Schwarzl, H., Stoll, B., Valavanoglou, A., and Wiedemann, M.: The THEMIS Fluxgate Magnetometer, Space Sci. Rev., 141, 235-264, 2008.

Balogh, A., Carr, C. M., Acuña, M. H., Dunlop, M. W., Beek, T. J., Brown, P., Fornacon, K.-H., Georgescu, E., Glassmeier, K.H., Harris, J., Musmann, G., Oddy, T., and Schwingenschuh, K.: The Cluster Magnetic Field Investigation: overview of in-flight performance and initial results, Ann. Geophys., 19, 1207-1217, 2001, http://www.ann-geophys.net/19/1207/2001/.

Bracewell, B. J.: The Fourier Transform and its Applications, 3rd ed. McGraw-Hill, Boston, 2000.

Capon, J.: High Resolution Frequency-Wavenumber Spectrum Analysis, Proc. IEEE, 57, 1408-1418, 1969.

Chanteur, G.: Spatial interpolation for four spacecraft: Theory, in Analysis Methods for Multi-Spacecraft Data, G. Paschmann and P. Daly (eds.), ISSI Scientific Reports Series, ESA/ISSI, Vol. 1, pp. 349-370, 1998.

Escoubet, C. P., Fehringer, M., and Goldstein, M.: The Cluster mission, Ann. Geophys., 19, 1197-1200, 2001, http://www.ann-geophys.net/19/1197/2001/.

Glassmeier, K.-H., Motschmann, U., and Schmidt, R. (Eds.): Data Analysis Tools, Proc. CLUSTER Workshop, ESA-SP 371, European Space Agency, Noordwijk, 1995.

Glassmeier, K.-H., Motschmann, U., Dunlop, M., Balogh, A., Acua, M. H., Carr, C., Musmann, G., Fornaçon, K.-H., Schweda, K., Vogt, J., Georgescu, E., and Buchert, S.: Cluster as a wave telescope - first results from the fluxgate magnetometer, Ann. Geophys., 19, 1439-1447, 2001 (Correction, 21, 1071, 2003).

Kirchner, J. W.: Aliasing in $1 / f^{\alpha}$ noise spectra: Origins, consequences, and remedies, Phys. Rev. E, 71, 066110, doi:10.1103/PhysRevE.71.066110, 2005.

Kittel, C.: Introduction to Solid State Physics, 7th ed., John Wiley \& Sons, New York, 1996.

Motschmann, U., Woodward, T. I., Glassmeier, K.-H., Southwood, D. J., and Pinçon, J. L.: Wavelength and direction filtering by magnetic measurements at satellite arrays: generalized minimum variance analysis, J. Geophys. Res., 101, 4961-4965, 1996.

Narita, Y., Glassmeier, K.-H., Schäfer, S., Motschmann, U., Sauer, K., Dandouras, I., Fornaçon, K.-H., Georgescu, E., and Rème, H.: Dispersion analysis of ULF waves in the foreshock using cluster data and the wave telescope technique, Geophys. Res. Lett., 30, SSC 43-1, doi:10.1029/2003GL017432, 2003.

Narita, Y. and K.-H. Glassmeier, Dispersion analysis of lowfrequency waves through the terrestrial bow shock, J. Geophys. Res., 110, A12215, doi:10.1029/2005JA011256, 2005.

Narita, Y., Glassmeier, K.-H., and Treumann, R. A.: Wave-number spectra and intermittency in the terres- 
trial foreshock region, Phys. Rev. Lett., 97, 191101, doi:10.1103/PhysRevLett.97.191101, 2006.

Narita, Y., Glassmeier, K.-H., Frnz, M., Nariyuki, Y., and Hada, T.: Observations of linear and nonlinear processes in the foreshock wave evolution, Nonlin. Processes Geophys., 14, 361-371, 2007, http://www.nonlin-processes-geophys.net/14/361/2007/.

Neubauer, F. and Glassmeier, K.-H.: Use of an array of satellites as a wave telescope, J. Geophys. Res., 95, 19115-19122, 1990.

Nyquist, H.: Certain topics in telegraph transmission theory, Amer. Inst. Elect. Eng., 47, 617-644, 1928.

Paschmann, G. and Daly, P. W. (Eds.): Analysis Methods for MultiSpacecraft Data, ISSI Scientific Report, SR-001, ESA Publications Division, Noordwijk, The Netherlands, 1998.

Paschmann, G. and Daly, P. W. (Eds.): Multi-Spacecraft Analysis Methods Revisited, ISSI Scientific Report, SR-008, ESA Communications, Noordwijk, The Netherlands, 2008.

Pinçon, J. L. and Lefeuvre, F.: Local characterization of homogeneous turbulence in a space plasma from simultaneous measurement of field components at several points in space, J. Geophys. Res., 96, 1789-1802, 1991.

Sahraoui, F.; Pinçon, J. L., Belmont, G., Rezeau, L., CornilleauWehrlin, N., Robert, P., Mellul, L., Bosqued, J. M., Balogh, A., Canu, P., and Chanteur, G.: ULF wave identification in the magnetosheath: The k-filtering technique applied to Cluster II data J. Geophys. Res., 108, SMP1-1, 1335, doi:10.1029/2002JA009587, 2003.
Sahraoui, F., Belmont, G., Rezeau, L., Cornilleau-Wehrlin, N., Pinçon, J. L., and Balogh, A.: Anisotropic turbulent spectra in the terrestrial magnetosheath as seen by the Cluster spacecraft, Phys. Rev. Lett., 96, 075002, doi:10.1103/PhysRevLett.96.075002, 2006.

Shannon, C. E.: Communication in the presence of noise, Proc. IRE, 37, 10-21, 1949.

Tjulin, A., Pinçon, J.-L., Sahraoui, F., André M., and CornilleauWehrlin, N.: The k-filtering technique applied to wave electric and magnetic field measurements from the Cluster satellites, J. Geophys. Res., 110, A11224, doi:10.1029/2005JA011125, 2005.

Unser, M.: Sampling - 50 years after Shannon, Proc. IEEE., 88, 569-587, 2000.

Vogt, J., Narita, Y., and Constantinescu, O. D.: The wave surveyor technique for fast plasma wave detection in multi-spacecraft data, Ann. Geophys., 26, 1699-1710, 2008, http://www.ann-geophys.net/26/1699/2008/. 